\title{
Altered Corticostriatal Connectivity and Exploration/Exploitation Imbalance Emerge as Intermediate Phenotypes for a Neonatal Dopamine Dysfunction
}

\author{
Barbara Y Braz ${ }^{*, 1}$, Gregorio L Galiñanes', ${ }^{1,3}$ Irene RE Taravini' ${ }^{2}$, Juan E Belforte ${ }^{1,4}$ and M Gustavo Murer ${ }^{1,4}$ \\ 'Systems Neuroscience Group, Institute of Physiology and Biophysics, IFIBIO 'Houssay', CONICET and University of Buenos Aires School of \\ Medicine, Buenos Aires, Argentina; ${ }^{2}$ Experimental Parkinsonism Lab, Institute of Pharmacology, School of Pharmacy and Biochemistry, University \\ of Buenos Aires, Buenos Aires, Argentina
}

\begin{abstract}
Findings showing that neonatal lesions of the forebrain dopaminergic system in rodents lead to juvenile locomotor hyperactivity and learning deficits have been taken as evidence of face validity for the attention deficit hyperactivity disorder. However, the core cognitive and physiological intermediate phenotypes underlying this rodent syndrome remain unknown. Here we show that early postnatal dopaminergic lesions cause long-lasting deficits in exploitation of shelter, social and nutritional resources, and an imbalanced exploratory behavior, where nondirected local exploration is exacerbated, whereas sophisticated search behaviors involving sequences of goal directed actions are degraded. Importantly, some behavioral deficits do not diminish after adolescence but instead worsen or mutate, particularly those related to the exploration of wide and spatially complex environments. The in vivo electrophysiological recordings and morphological reconstructions of striatal medium spiny neurons reveal corticostriatal alterations associated to the behavioral phenotype. More specifically, an attenuation of corticostriatal functional connectivity, affecting medial prefrontal inputs more markedly than cingulate and motor inputs, is accompanied by a contraction of the dendritic arbor of striatal projection neurons in this animal model. Thus, dopaminergic neurons are essential during postnatal development for the functional and structural maturation of corticostriatal connections. From a bottom-up viewpoint, our findings suggest that neuropsychiatric conditions presumably linked to developmental alterations of the dopaminergic system should be evaluated for deficits in foraging decision making, alterations in the recruitment of corticostriatal circuits during foraging tasks, and structural disorganization of the frontostriatal connections.

Neuropsychopharmacology (20I5) 40, 2576-2587; doi:I0.I 038/npp.20 I5.I04; published online I 3 May 2015
\end{abstract}

\section{INTRODUCTION}

Early postnatal disturbances of forebrain dopamine (DA) neurotransmission are relevant to our understanding of neuropsychiatric conditions like the attention deficit hyperactivity disorder (ADHD) (Biederman and Faraone, 2005; Swanson et al, 2007). Patients diagnosed with ADHD usually show hyperactivity, impulsivity, and inattention, but the clinical presentation of ADHD is highly heterogeneous. This heterogeneity relates to the main symptoms and also to the high rates of comorbidity with mood, impulse control, and personality disorders. Symptoms usually diminish after

*Correspondence: BY Braz, Systems Neuroscience Group, Institute of Physiology and Biophysics, IFIBIO 'Houssay', CONICET and University of Buenos Aires School of Medicine, 2155 Paraguay Street, 1121 Buenos Aires, Argentina, Tel/Fax: 54(I I)5950-9500 (ext 2137),

E-mail: barbybraz@gmail.com or lab.fcn@gmail.com

${ }^{3}$ Current address: Department of Fundamental Neuroscience, University of Geneva, CMU, Rue Michel Servet 1, 1211 Geneva 4, Switzerland.

${ }^{4}$ The last two authors contributed equally to this work.

Received 30 October 2014; revised 13 March 20 I5; accepted 27 March 2015; accepted article preview online 15 April 2015 adolescence, but children diagnosed with ADHD show significant psychosocial and educational impairments as adults and significant comorbidities through their lifespan (Biederman and Faraone, 2005; Wilens et al, 2009; Klein et al, 2012). Although patients with ADHD show depressed DA activity in the caudate nucleus (Volkow et al, 2007), delayed maturation of caudate volume (Castellanos and Tannock, 2002), and respond to DA neurotransmissionenhancing drugs (Biederman and Faraone, 2005), it is unclear how early dopaminergic alterations relate to symptoms. Findings showing that neonatal forebrain dopaminergic depletion leads to hyperactivity and learning deficits in rodents suggested that early dopaminergic manipulations may be useful to study behavioral phenotypes relevant to ADHD and related disorders (Shaywitz et al, 1976; Archer et al, 1988; Moy, 1995; Avale et al, 2004). However, the core cognitive alterations underlying this rodent syndrome remain unknown. Moreover, there is little evidence that any of the purported animal models of ADHD replicate the alterations of corticostriatal connectivity observed in patients.

Here we have reexamined the behavioral effects of a dopaminergic neuron (DAN) lesion induced by 6-hydroxydopamine 
(6-OHDA) in mice neonates, a purported model of ADHD, from a bottom-up perspective. In line with current views emphasizing the role of DA signals in orienting behavior toward the discovery of information (Bromberg-Martin and Hikosaka, 2009; Dolan and Dayan, 2013; Pearson and Platt, 2013), we focused on the exploration and exploitation of information-rich novel environments rather than on conditions of instrumental learning favoring model-free cognitive control (Dolan and Dayan, 2013). As information is rewarding by itself (Bromberg-Martin and Hikosaka, 2009), we speculate that developing without DANs leads to the acquisition of an imbalanced exploration/exploitation strategy, with enduring consequences on behavior. Moreover, because studies in children and adults with ADHD report structural and functional deficits in the associative corticostriatal circuits (Konrad and Eickhoff, 2010; Liston et al, 2011), and experimental evidence supporting that postnatal DA availability shapes corticostriatal connectivity is scarce (Galiñanes et al, 2009), we have performed in vivo electrophysiological studies of corticostriatal circuits and reconstructed the dendritic arbors of striatal neurons in neonatally DAN-lesioned mice.

\section{MATERIALS AND METHODS}

Further information is available online in Supplementary Experimental Procedures.

\section{Animals}

CF-1 mice were used for behavioral and electrophysiological recordings and Drd1a-tomato/Drd2-eGFP double transgenic mice (Shuen et al, 2008) for morphological reconstructions of neurons. Mice were maintained under a $12: 12 \mathrm{~h}$ light/ dark cycle with ad libitum food and water. All experimental procedures were in accordance with the institutional (IACUC of the University of Buenos Aires School of Medicine) and government regulations (SENASA RS617/2002, Argentina).

\section{Lesions}

Bilateral DAN lesion was performed at PD2 following published protocols (Galiñanes et al, 2009). Pups received injections of 6-OHDA or vehicle in each lateral ventricle, after desipramine pretreatment, under hypothermal anesthesia. Control and DAN-lesioned mice were housed together.

\section{Behavioral Tests}

DAN-lesioned and age-matched control mice (see Results) were subjected to the following tests: standard open field, activity in a small arena, marble burying, elevated plus maze, large open field, Y maze spontaneous alternation, hoarding behavior, social interaction, and nest-building test as previously described (Belforte et al, 2010) with some modifications. All findings were confirmed in at least two separate cohorts of animals.

\section{In Vivo Electrophysiology}

Striatal recordings were performed with 24-channel two-shank or 32-channel four-shank silicon probes under urethane anesthesia with the animal fixed to a stereotaxic frame (Galiñanes et al, 2009, 2011). Cortical electrical stimulation was applied through concentric bipolar electrodes placed into prelimbic (Prl), cingulate, and motor areas of the frontal cortex. Field potentials were analyzed offline with custommade Matlab routines.

\section{Morphological Analysis}

Fixed brain slices $(200 \mu \mathrm{m}$ thick) were used for iontophoretical neuronal labeling with Lucifer yellow (LY) following published protocols (Suárez et al, 2014). After injections, LY was detected using colorimetric immunohistochemistry. Morphological reconstructions were made under transmitted light at $\times 100$ using Mercator software.

\section{Histology and Immunohistochemistry}

Electrode location was histologically verified as in Galiñanes et al (2011). Striatal depletion of tyrosine hydroxylase (TH) immunoreactive terminals was quantified as described in Galiñanes et al (2009).

\section{Statistical Analysis}

Statistical analysis was performed using SigmaPlot 11.0 or InfoStat 2014.

\section{RESULTS}

\section{Setting-Dependent Alteration of Exploratory Behavior after Neonatal DAN Lesion}

Early dopaminergic lesions produce juvenile locomotor hyperactivity that wanes in adults, presumably because compensatory mechanisms are called into play (Shaywitz et al, 1976; Avale et al, 2004; Galiñanes et al, 2009). However, certain forms of hyperactivity can persist in the adult, especially after extensive dopaminergic denervation (Miller et al, 1981; Luthman et al, 1997). Hyperactivity and repetitive behaviors distinctive of human neuropsychiatric disorders may emerge only in specific settings, and symptoms observed in juvenile patients can metamorphose and persist in the adult. Therefore, we asked whether adult mice that were DAN lesioned as neonates exhibit hyperactivity and repetitive behaviors under the appropriate conditions.

DAN-lesioned and age-matched control animals were subjected to a battery of behavioral tests after reaching adulthood ( $>12$ weeks old). As expected, when tested in an open field arena of standard size $(40 \times 40 \mathrm{~cm})$, DAN-lesioned mice did not show hyperactivity (Figure 1a). However, in a smaller setting designed to score repetitive behaviors, they displayed increased vertical exploratory activity (RM-ANOVA, $P<0.001$; Figure $1 \mathrm{~b}$ and Supplementary Movies S1 and S2). To assess self-oriented perseveration, mice were sprayed with water mist to induce grooming. Induced grooming was reduced in DAN-lesioned mice (RM-ANOVA, $P<0.001$; Figure 1b). Moreover, in the marble-burying test, which evaluates object-oriented perseveration, they did not show interest in burying marbles (RM-ANOVA, $P<0.001$; Figure 1c and Supplementary Movies S3 and S4). The expression of repetitive behaviors may be modified by anxiety; 
a

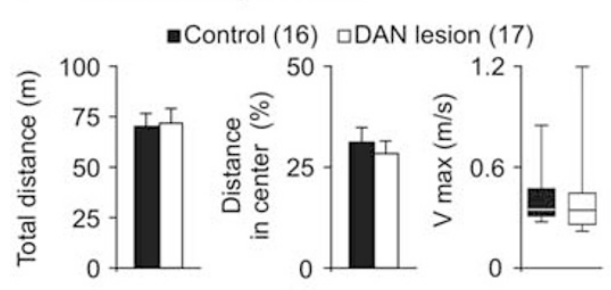

b Small Arena

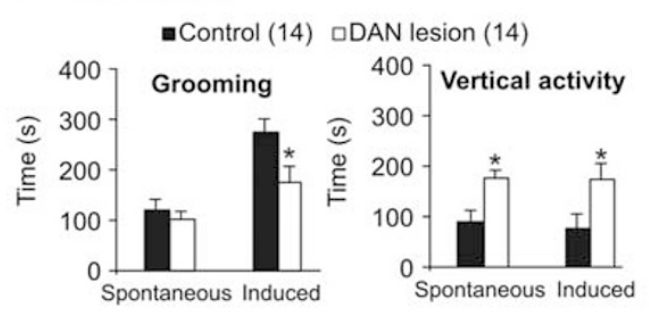

C Marble Burying

- Control (16) aDAN lesion (17)
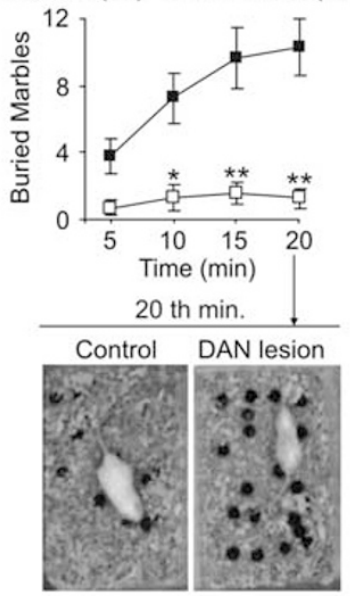

\section{d Elevated Plus Maze}

\section{- Control (22) aDAN lesion (22)}
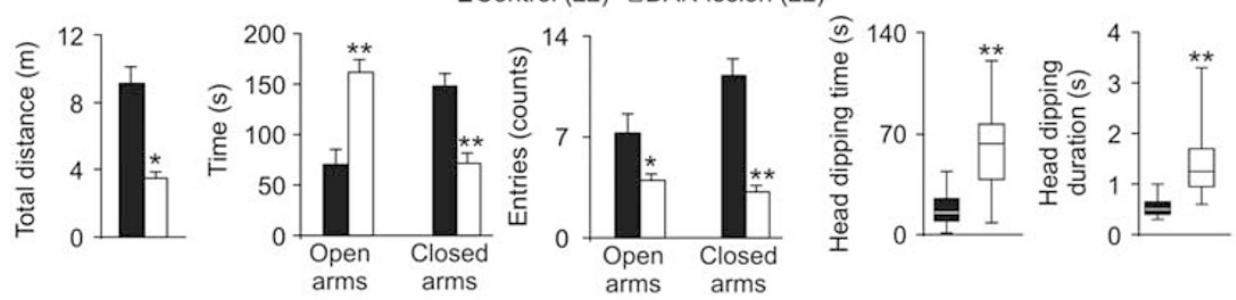

f Y maze

e Large Open Field aControl (8) 口DAN lesion (8)
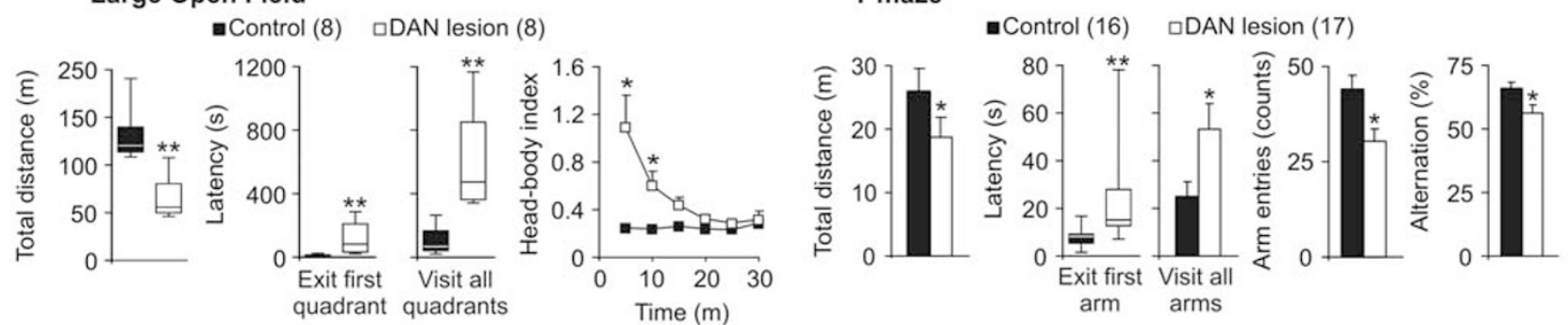

Figure I Setting-dependent alteration of exploratory behavior in adulthood after neonatal DAN lesion. (a) Standard open field. No difference was observed in total traveled distance ( $t$-test, $\left.t_{31}=-0.16, P=0.9\right)$, distance in the center ( $t$-test, $\left.t_{31}=0.6, P=0.6\right)$, and maximal velocity (Mann-Whitney $U=|| \mid 6$, $P=0.5$ ). (b) Small arena. DAN-lesioned mice showed normal grooming but lower grooming induction (RM ANOVA, interaction: $F_{I, 55}=7, P=0.01, * P<0.05$ Tukey's post hoc test). Vertical activity was increased in both conditions (RM ANOVA, treatment effect: $F_{1.55}=\mid 5$, *P $<0.00 I$ ). (c) Marble burying. DANlesioned mice showed lower levels of burying across time (RM ANOVA, interaction: $F_{6,131}=8.5, P<0.00$ I, Tukey's post hoc test $* P<0.05$, *** $P<0.00 \mathrm{I}$ ). Representative pictures at the end of the test are shown. (d) Elevated plus maze. DAN-lesioned mice traveled less along the maze (Mann-Whitney $U=53$, $* * *<0.00 \mathrm{I}$ ), spent more time in the open arms (RM ANOVA, interaction: $F_{1,87}=26, P<0.00$ I, Tukey's post hoc test $* * P<0.00 \mathrm{I}$ ), entered fewer times to the maze arms (RM ANOVA, interaction: $F_{1,87}=11, P=0.002$, Tukey's post hoc test $* P<0.05 * * P<0.00 I$ ), and exhibited more (Mann-Whitney $U=58$, $* * P<0.00 \mathrm{I}$ ) and longer head dippings (Mann-Whitney $U=20$, $* * P<0.00 \mathrm{I}$ ). (e) Large arena. Total distance traveled was lower (Mann-Whitney $U=0$, $* * P<0.00$ I) whereas latency to exit the first quadrant (Mann-Whitney $U=I$, $* * P<0.00 \mathrm{I}$ ) and to visit all quadrants was higher for the lesioned mice (MannWhitney $U=0$, $* * P<0.00$ I). Right: DAN-lesioned mice showed increased local exploration in the form of a higher distance traveled by the head relative to the body (RM ANOVA, interaction: $F_{5,89}=9, P<0.00$ I, Tukey's post hoc test $* P<0.05, * * P<0.00$ I). (f) Spontaneous alternation in $Y$ maze. Total distance traveled in $Y$ maze was lower ( $t$-test, $t_{3 \mid}=2.7, * P=0.0 \mathrm{I}$ ), whereas latency to leave the first arm (Mann-Whitney $U=25$, ** $P<0.00 \mathrm{I}$ ) and to visit all arms (Mann-Whitney $U=76$, *P=0.032) was longer in lesioned than control mice. Moreover, number of entries to arms (Mann-Whitney $U=59$, *P=0.005) and alternation ( $t$-test, $t_{31}=2.4, * P=0.02$ ) were lower in lesioned mice. Data are mean $\pm S E M$, or median \pm interquartile ranges and I0-90th percentiles; animal numbers are indicated in parentheses.

however, anxiety-related measures in the open field were normal in DAN-lesioned mice (Figure 1a). Moreover, their phenotype in the elevated plus maze was not typical of changes in anxiety levels (Figure 1d). DAN-lesioned mice entered fewer times to the open than the closed arms of an elevated plus maze compared to control mice (RM-ANOVA, $P=0.002$ ), but spent more time in the open arms (RMANOVA, $P<0.001)$ displaying increased local exploratory behaviors (head dippings, Mann-Whitney test, $P<0.001$; Figure 1d). Thus, although the influence of anxiety in certain phenotypes cannot be completely excluded, we favor the view that DAN-lesioned mice experience an abnormal mode of exploratory behavior rather than locomotor hyperactivity or stereotyped repetitive behaviors.
To further evaluate the effect of the setting on exploratory behavior, we exposed DAN-lesioned mice to a large-size open arena (1.25 $\mathrm{m}$ in diameter) where control mice exhibit a compound strategy involving wide-field ambulation and local exploration based on swaying head movements (Supplementary Movies S5 and S6). In this setting, DANlesioned animals take 10 times longer to leave the starting quadrant and five times longer to visit the four quadrants of the arena than control mice (Mann-Whitney test, $P<0.001$; Figure 1e). Moreover, the total distance traveled was markedly reduced in DAN-lesioned mice (Mann-Whitney test, $P<0.001$; Figure 1e). Conversely, local exploration achieved by continuous swaying head movements was significantly increased (RM-ANOVA, $P<0.001$; 
a Social interaction test

- Control (11) aDAN lesion (13)

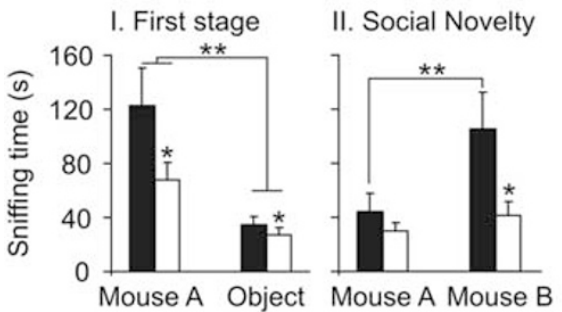

C Hoarding behavior

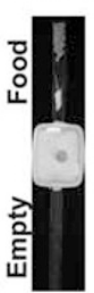

$$
\text { - Control (7) }
$$

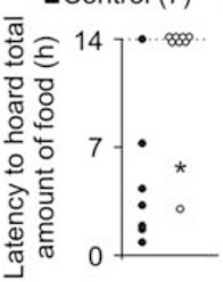

b Nesting

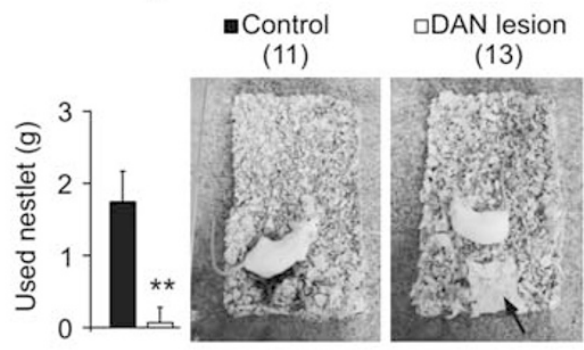

Figure 2 Reduced exploitation in neonatally DAN-depleted animals when tested as adults. (a) Social interaction test. DAN-depleted mice showed lower sniffing levels directed to the unfamiliar mouse A (left; RM ANOVA, treatment factor: $F_{1,47}=4.5, * P=0.046$, chamber factor: $F_{1,47}=23, * * P<0.00 I$ ) and failed to show an increase in social approach toward a novel conspecific (mouse B) (right; RM ANOVA, interaction: $F_{1,47}=8.9, \mathrm{df}=1, P=0.007$; Tukey's post hoc test $* P<0.05$, $* * P<0.00 \mathrm{I}$ ). (b) Nesting. DAN-depleted mice used less material from the cotton nestlet (arrow) provided for the test $\left(t\right.$-test, $t_{23}=3.6$, $* * P=0.00$ I). (c) Hoarding behavior. Photo of the behavioral setup: modified home cage connected with two $50 \mathrm{~cm}$ long tubes (see Results). From left to right: DAN-depleted mice hoarded less food ( $t$-test, $t_{13}=3.1$, * $P=0.009$ ), took longer to hoard all available pellets $($ Mann-Whitney $U=7.5$, *P=0.0 I), and visited fewer times the food and empty tubes (RM ANOVA, treatment effect: $F_{1,29}=43, * P<0.00$ I), but spent the same time in each tube than control mice (RM ANOVA). Data are mean \pm SEM; animal numbers are indicated in parentheses.

Figure 1e and Supplementary Movie S6), suggesting an imbalanced exploratory strategy.

To further study this possibility we exposed the mice to a $\mathrm{Y}$ maze, a test assessing the natural tendency of mice to alternate their choice of maze arms in order to maximize information gathering in this environment (Figure 1f). DAN-lesioned animals visited less times the maze arms (Mann-Whitney test, $P=0.005$ ), stayed for longer in the first visited arm ( $t$-test, $P=0.01)$, and took longer to visit all three arms (Mann-Whitney test, $P=0.032$ ). The $\mathrm{Y}$ maze also revealed a deficient spontaneous alternation (sequential exploration of the three arms). Although reliable alternation was observed in control animals, DAN-lesioned mice displayed a significant reduction to chance level ( $t$-test, $P=0.02$; Figure 1f). Although this would be interpreted as a working memory deficit, here it could relate to the higher memory load resulting from the longer time the mice spent in each arm. Thus, the neonatal dopaminergic lesion produces pervasive alterations of exploratory behavior in settings where costly search strategies are required to gather information.

\section{Reduced Exploitation of Opportunities in DAN-Lesioned Animals}

Optimal utilization of environmental opportunities, including social, nutritional, and shelter resources, depends on foraging decision making (Cohen et al, 2007; Adams et al, 2012). Foraging decisions involve choosing between exploiting a local resource and searching for alternative resources in other places. DAN-lesioned animals may be impaired at making foraging decisions, as they fail to bury strange objects, to seek refuge in risky environments, and to adaptively explore large arenas and maze arms (Figure 1). To investigate whether DAN-lesioned animals are impaired at exploiting primary resources, we studied their performance in a series of ethologically based tests assessing social, shelter, and nutritional behaviors spontaneously emitted by mice (Deacon, 2012).

In a three-chamber social interaction test that evaluates the natural tendency of mice to explore a novel conspecific over a novel object, DAN-lesioned mice show a significant decrease in social approach behavior directed to the mouse (RM-ANOVA, interaction: $P=0.088$, treatment: $P=0.046$, chamber: $P<0.001$; Figure 2a). Moreover, they fail to show an increase in social approach toward a novel conspecific when paired with a familiar one during the second part of the test (Figure 2a; RM-ANOVA, interaction: $P=0.007$ ). Thus, DAN-lesioned mice exhibit deficits in social exploratory behavior and at discriminating social novelty.

Nest-building behavior is connected with exploitation of natural resources to secure shelter, facilitate heat conservation, and promote social behaviors (Deacon, 2012). To minimize the effect of exploration deficiencies, this test was conducted in the mouse home cage. Most control mice formed a nest using a cotton nestlet provided as starting material the evening before, but DAN-lesioned mice had not built clear nests by the following morning ( $t$-test, $P<0.001$; Figure $2 \mathrm{~b}$ ), consistently with the idea of decreased exploitation of primary resources. 
To directly assess exploration/exploitation balance we adapted a test that examines hoarding behavior (Deacon, 2012) to increase exploration load (Figure 2c). Mice were individually housed in a modified home cage that connects with two $50 \mathrm{~cm}$ long wire mesh tubes extending in opposite directions. After $24 \mathrm{~h}$ of habituation to the central cage, animals were allowed to freely explore the tubes during the entire night under infrared video surveillance. One tube contained $100 \mathrm{~g}$ of regular food pellets and the other one was empty. Control mice hoarded most of the food during the first $4 \mathrm{~h}$, whereas the DAN-lesioned mice hoarded on average $36 \pm 13 \mathrm{~g}$ along the $14 \mathrm{~h}$ of the test $(t$-test, $P<0.05)$. Moreover, control and DAN-lesioned mice spent the same time in the loaded and unloaded tubes (RM-ANOVA), indicating that the DAN-lesioned animals make a less efficient use of their time in the loaded tube. Finally, control animals entered more times to the unloaded tube than DAN-lesioned mice, thus being more efficient in updating cognitive maps of the environment.

Overall, in adulthood, neonatally DAN-lesioned animals are impaired at foraging for information and at exploiting primary environmental resources (Supplementary Figure S1).

\section{Exploration/Exploitation Deficits Emerge Early and Worsen with Age}

As previous studies have not examined the exploration/ exploitation phenotype induced by neonatal DAN lesions so extensively, we asked whether the deficits that we observe in the adult emerge during the juvenile period. Juvenile mice (30-50 days old) neonatally depleted of DANs show exacerbated vertical exploration ( $t$-test, $P=0.004$; Figure 3a) and deficits in exploitation as assessed in the marble-burying and nest-building tests (RM-ANOVA, $P<0.001$; Figure $3 \mathrm{~b}$ and $\mathrm{c}$ ). A subtle increase in local exploration was also observed in the large circular arena, but globally, juvenile mice exhibited normal wide-field exploration in this setting (Figure 3d). In contrast, the cognitively more demanding $\mathrm{Y}$ maze revealed navigation deficits qualitatively similar to those observed in adults (Figure 3e). Remarkably, at difference with the adults (Figure 1e), alternation in the $\mathrm{Y}$ maze was preserved in juvenile mice ( $t$-test, $P=0.1$, Figure $3 \mathrm{e}$ ). This dissociation observed in juvenile mice, of alternation performance from navigation deficits, suggests that the deficient alternation observed in adults does represent a working memory deficit. Finally, in the modified hoarding behavior test, juvenile mice showed deficits qualitatively similar to those observed in adults (Figure 3f). Thus, alterations in the exploration/exploitation balance emerge early, but symptoms persist and worsen in adults.

\section{Reduced Prefrontostriatal Functional Connectivity in DAN-Lesioned Animals}

Several factors suggested an involvement of associative corticostriatal circuits in the behavioral deficits observed in DAN-lesioned mice. First, this animal model shows maximal depletion of dopaminergic nerve terminals in the medial part of the dorsal striatum and less compromise of the dorsolateral striatum and nucleus accumbens (Galiñanes et al, 2009). Second, foraging decisions involve neuronal activity in associative corticostriatal circuits (Daw et al, 2006; Hayden
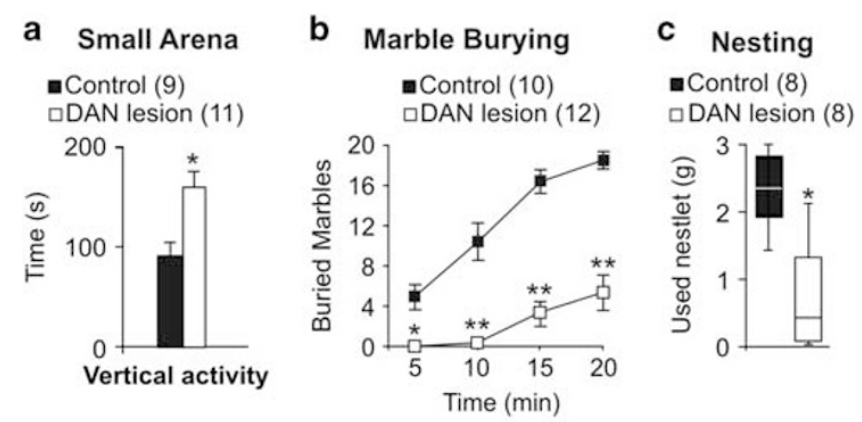

d Large Open Field

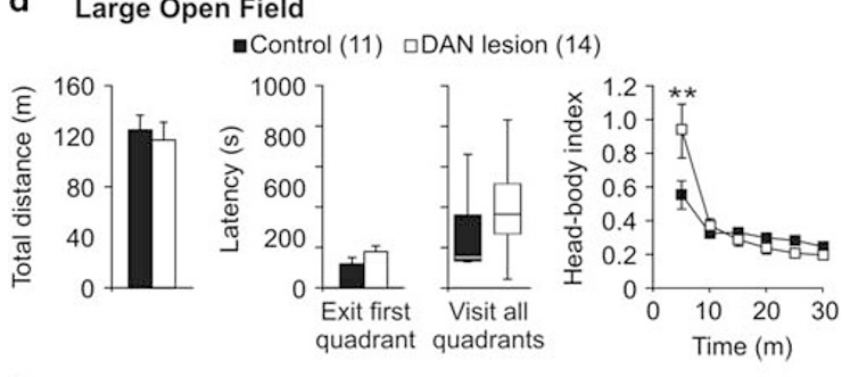

e Y maze
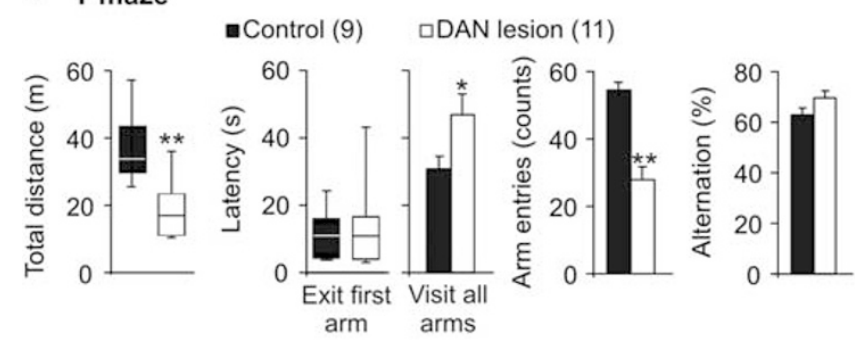

f Hoarding behavior aControl (8) 口DAN lesion (8)
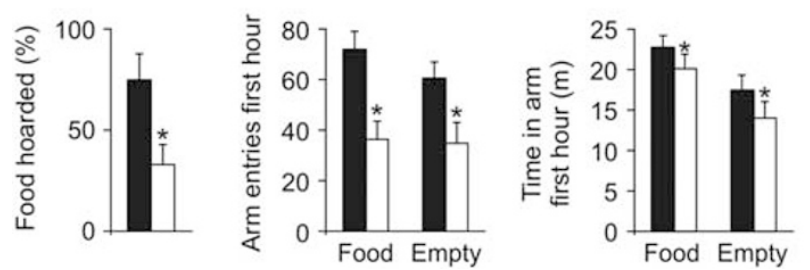

Figure 3 Exploration/exploitation deficits emerge in juvenile animals. (a) Small arena. DAN-lesioned mice showed an increase in spontaneous vertical activity ( $t$-test, $t_{18}=-3.2$, $* P=0.004$ ). (b) Marble burying. DAN-depleted mice showed lower levels of burying across time (RM ANOVA, interaction: $F_{3,87}=9.9, P<0.001$, Tukey's post hoc test $* P<0.05$, ** $P<0.00$ I). (c) Nesting. DAN-lesioned mice used less material from the cotton nestlet provided for the test (Mann-Whitney, $U=3$, ${ }^{*} P=0.001$ ). (d) Large arena. No differences were observed in total traveled distance ( $t$-test, $\left.t_{23}=0.4, P=0.7\right)$, latency to exit the first quadrant (t-test, $t_{23}=-1.3, P=0.2$ ), or latency to visit all quadrants (Mann-Whitney, $U=47, P=0.1$ ). DAN-lesioned mice showed increased local exploration in the first $5 \mathrm{~min}$ of the test (RM ANOVA, interaction: $F_{5,89}=5.3, P<0.001$, Tukey's post hoc test $\left.* P<0.05\right)$. (e) $Y$ maze. Total distance traveled was lower (Mann-Whitney, $U=7$, ** $P<0.001$ ) and latency to visit all arms longer ( $t$-test, $t_{18}=-2.1$, * $P=0.047$ ) in DAN-lesioned than control mice. The number of entries to arms were lower in lesioned mice ( $t$-test, $t_{19}=5.6, P<0.00 \mathrm{I}$ ), and there was no difference in alternation between groups ( $t$-test, $t_{19}=-1.7, P=0.1$ ). (f) Hoarding behavior. From left to right: DAN-depleted mice hoarded less food ( $t$-test, $t_{13}=3.1$, $\left.* P=0.009\right)$ and visited fewer times both tubes (RM ANOVA, treatment effect: $F_{1,31}=9.9$, $* P=0.007$, tube effect: $F_{1,31}=6.7, P=0.02$ ). The time spent in the tubes was lower in lesioned mice and both groups preferred the food arm (RM ANOVA, treatment effect: $F_{\mid, 31}=10.6$, ${ }^{*} P=0.006$, tube effect: $F_{\mid, 31}=5.8, P=0.031$ ). Data are mean $\pm \mathrm{SEM}$, or median \pm interquartile ranges and $10-90$ th percentiles animal numbers are indicated in parentheses. 
et al, 2011). Third, patients with ADHD and related disorders show functional alterations in the associative corticostriatal circuits (Konrad and Eickhoff, 2010; Liston et al, 2011).

To assess corticostriatal functional connectivity in vivo, we extracellularly recorded the striatal response to Prl stimulation through a multisite electrode located in the dorsomedial striatum, where $\operatorname{Prl}$ stimulation evokes high-amplitude responses in the anesthetized mouse (Galiñanes et al, 2011). Prl stimulation evokes a well-characterized striatal field wave (Figure 4a) whose amplitude is linearly related to that of depolarizing postsynaptic potentials and population spike responses of medium spiny projection neurons (MSNs) (Galiñanes et al, 2011). Recordings from adults showed smaller responses in DAN-lesioned mice, as shown by a significant leftward shift of the frequency distribution of field wave amplitudes (Kolmogorov-Smirnov test, $P<0.001$ ) and by a $42 \%$ reduction of the maximal response attained within the more responsive striatal area (RM-ANOVA, $P<0.001$; Figure $4 \mathrm{~b}$ ). Juvenile mice showed less marked alterations. There was a small but significant leftward shift in the distribution of response amplitudes (Kolmogorov-Smirnov test, $P<0.001$ ), but maximal field responses did not significantly differ between DAN-lesioned and control mice (Figure $4 \mathrm{c}$ and $\mathrm{d}$ ). Besides, although juvenile mice show depressed population spike responses to Prl stimulation, the population spike response is more markedly depressed in adults (Supplementary Figure S2). To determine whether reduced occupancy of DA receptors (DARs) during the recordings can per se induce functional deficits similar to those induced by neonatal DAN lesions, we assessed the effect of DAR antagonists on striatal responses in control mice. Acute DAR blockade did not modify the striatal field and population spike response in adult control animals (Figure 4e and Supplementary Figure S2). Thus, the neonatal DAN lesion induces early alterations of prefrontostriatal functional connectivity that evolve into a marked reduction in functional connectivity in the adult.

Studies in rodents (Kasanetz et al, 2008; Mailly et al, 2013) and primates (Haber and Knutson, 2010) show that functionally related corticostriatal projections partially overlap. To assess whether neonatal DAN depletion affects corticostriatal integration that could be crucial for decision making (Haber and Knutson, 2010), we placed a multisite electrode array (32 sites in 4 shanks) spanning most of the dorsal striatum to record responses to electrical stimulation of Prl, cingulate, and motor cortices in control and DAlesioned mice under anesthesia (Figure 5a). In control mice, $\mathrm{Prl}$ and cingulate stimulation evokes reliable responses in the three more medial shanks with a topography that recapitulates rodent anatomy of corticostriatal projections (Figure 5b and c); (Mailly et al, 2013). Conversely, responses to motor cortex stimulation are maximal in the lateral shank and negligible in the medial one. This organized pattern is degraded in DAN-lesioned mice that show attenuated responses to Prl stimulation across the whole span of the Prl projection field and a distorted striatal representation of cingulate and motor cortex inputs (Figure 5d).

\section{Contraction of Medium Spiny Neuron Dendritic Fields in Neonatally DAN-Lesioned Animals}

The more marked attenuation of prefrontostriatal connectivity observed in adult than juvenile mice suggested that the lesion induces progressive corticostriatal alterations. During adolescence, the volume of the human caudate nucleus decreases (Castellanos and Tannock, 2002) through a process that is regulated by the DA transporter and D4 receptor genes (Durston et al, 2005) and that has been speculatively attributed to synaptic pruning (Toga et al, 2006). Consequently, we looked for a morphological correlate of the diminished functional connectivity observed in adult DANlesioned mice.

We used BAC-transgenic mice that allow visualizing direct and indirect pathway MSNs (dMSNs and iMSNs) (Shuen et al, 2008) to label and subsequently reconstruct, using an image analysis system, MSNs located in the dorsomedial striatum (Figure 6a), where depletion of dopaminergic terminals is maximal in this animal model (Figure 6b). The number of primary dendrites, of branching points in the dendrites, and the cell body area, were not affected by the DAN lesion (Supplementary Figure S3). However, MSNs showed a significant reduction of total dendritic length in DAN-lesioned mice (Figure 6c; two-way ANOVA, $P<0.05$ for treatment), without changes in spine density (Figure $6 \mathrm{~d}$ ). Sholl analysis of dendritic arbors revealed that iMSN dendrites located $>55 \mu \mathrm{m}$ away from the soma consistently show reduced length and fewer intersections in DANlesioned mice (RM-ANOVA interactions, $P<0.001$; Figure 6e). Fewer intersections were also detected in dMSNs (RM-ANOVA interaction, $P=0.027$ ), but the Sholl analysis did not reveal changes in dendritic length, suggesting less robust effects of the DAN lesion in dMSNs (Figure 6e). Thus, the span of the dendritic arbor of MSNs is reduced in neonatally DAN-lesioned animals, more markedly in iMSNs than dMSNs.

\section{DISCUSSION}

Animals facing novel environments have to decide whether to exploit what is immediately available or to search new locations (Adams et al, 2012; Cohen et al, 2007). Search processes may involve sophisticated sequences of goaldirected actions relying on mechanisms of cognitive control comparable to those involved in exploitation (Cohen et al, 2007; Daw et al, 2006; Hayden et al, 2011). Moreover, recent studies have shown that information is rewarding for animals and that DANs encode advanced information about rewards as if it were a primary reward (Bromberg-Martin and Hikosaka, 2009). Thus, exploitation and search seem to share a common drive, the salience of primary (eg, food) and abstract (eg, information) rewards (Pearson and Platt, 2013). Thus, DAN-lesioned mice seem to have a general deficiency in processing salience, as they fail both to exploit and forage for information in novel settings. This is best seen in a hoarding behavior test adapted to increase information needs. Control mice were more efficient at hoarding food and also at updating information about the environment than DAN-lesioned mice. Although the ultimate mechanisms underlying deficient salience processing in this animal model remain uncertain, DAN-lesioned animals exhibit a 
a

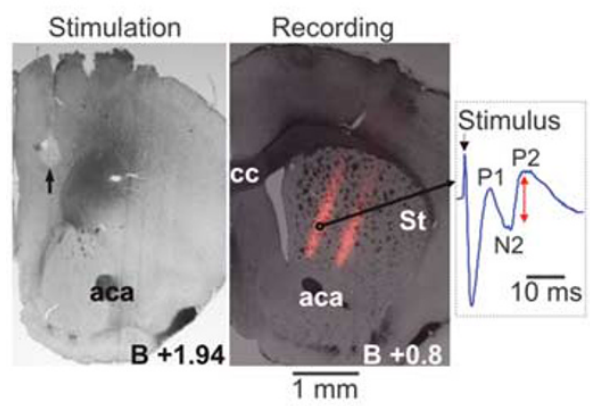

d

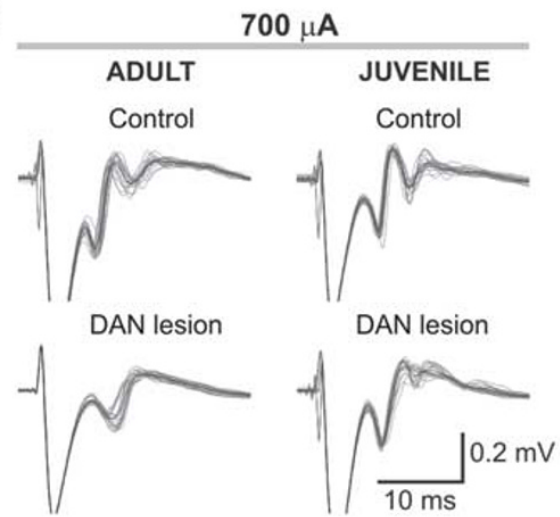

e

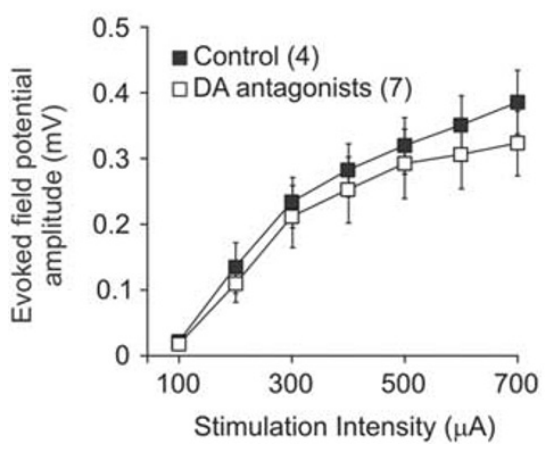

b

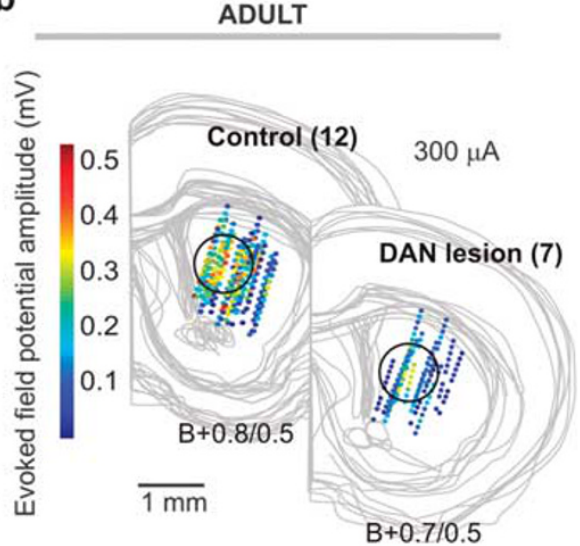

ADULT

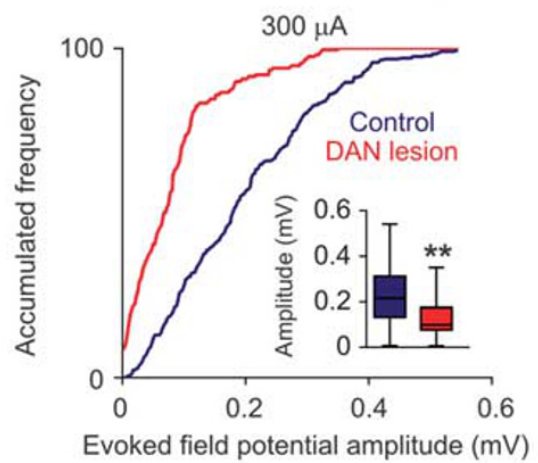

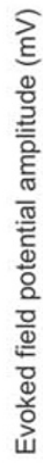
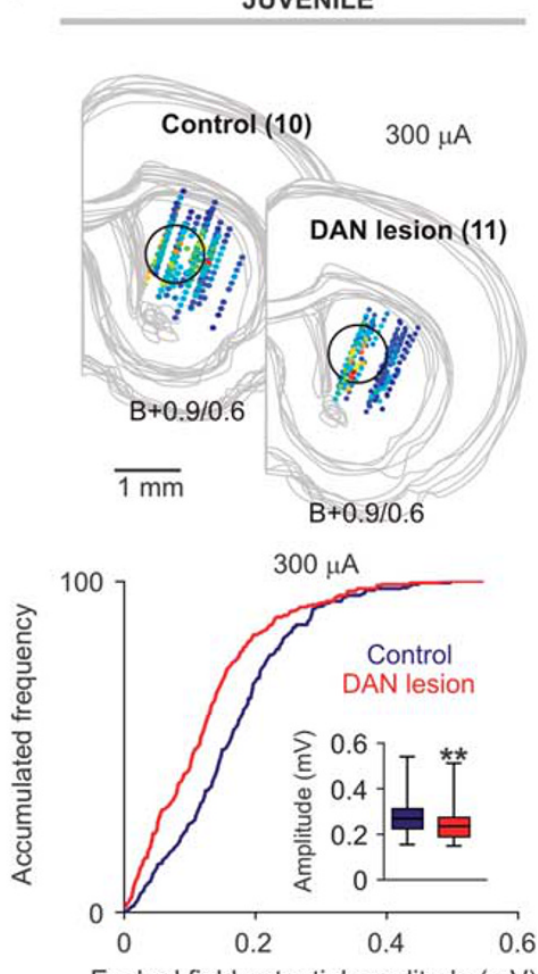

Evoked field potential amplitude $(\mathrm{mV})$

Figure 4 Functional corticostriatal disconnection in neonatally dopamine depleted animals. (a) Representative histological sections showing the location of cortical stimulation (left) and striatal recording (right) electrodes and representative trace of a striatal response evoked by cortical stimulation (aca, anterior commissure; St, striatum; cc, corpus callosum). Field potential amplitude was measured between the N2 and P2 peaks. Arrow points to location of the stimulating electrode. (b) Corticostriatal connectivity in adults. Top: Topographical reconstruction of recording sites. Colors represent the average field potential amplitude evoked by a cortical stimulation intensity of $300 \mu \mathrm{A}$. Middle: Cumulative frequency distribution and box and whisker plots (median with interquartile ranges and 10-90th percentiles) of evoked field potential amplitudes induced by $300 \mu \mathrm{A}$ stimuli applied to the prelimbic cortex (KolmogorovSmirnov test $* * P<0.00 \mathrm{I}$ ). Bottom: Amplitude of the striatal field response (averaged for channels within the circle shown at the top) as a function of

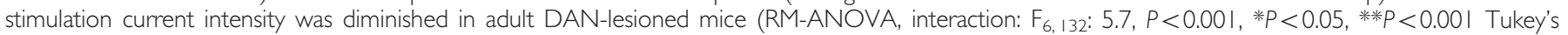
post hoc test). (c) Corticostriatal connectivity in juvenile mice. Top, middle, and bottom panels as in (b). Middle: ** $P<0.00$ I (Kolmogorov-Smirnov test). (d) Representative traces of maximal local field potential responses (20 individual trials overlapped in gray, average trace in black). (e) Amplitude of the striatal field response was not affected by DA receptor antagonists (eticlopride $0.25 \mathrm{mg} / \mathrm{kg}$ and SCH $233900.25 \mathrm{mg} / \mathrm{kg}$, i.p.) (RM ANOVA). Data are mean \pm SEM, or median \pm interquartile ranges and 10-90th percentiles animal numbers are indicated in parentheses.

disorganized corticostriatal functional topography affecting more markedly projections from medial prefrontal cortex than those from lateral frontal areas. The more prominent functional connectivity alterations observed in adult than juvenile mice, and the atrophy observed in the dendrites of MSNs, support that corticostriatal connectivity is shaped by DANs during postnatal development (Galiñanes et al, 2009).
Neonatal DAN depletion has been evaluated as an ADHD model from a top-down perspective. In addition to hyperactivity, this rodent model exhibits learning deficits in tasks with dissimilar neural substrates such as lever-pressing operant conditioning (Heffner and Seiden, 1983), active avoidance learning (Smith et al, 1973), and the Morris water maze (Archer et al, 1988). Remarkably, learning is not 
a

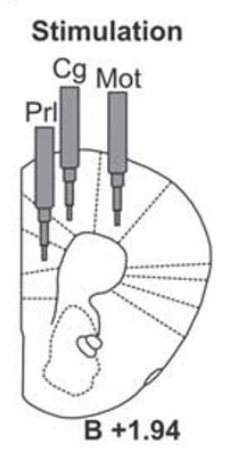

Recording

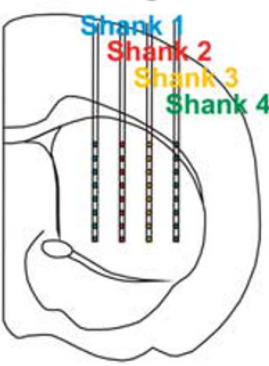

$B+1.0 /+0.6$ b

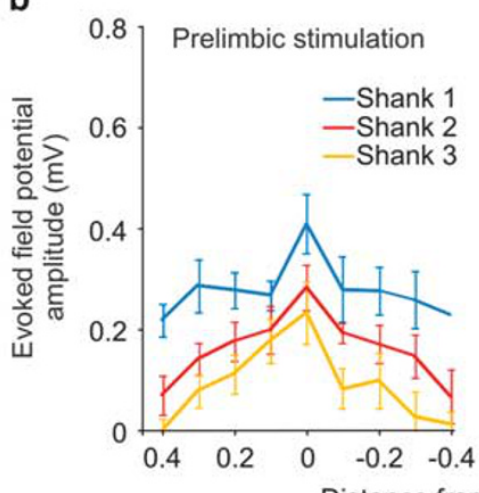

Control (8)

Cingulate stimulation $\quad$ Motor stimulation

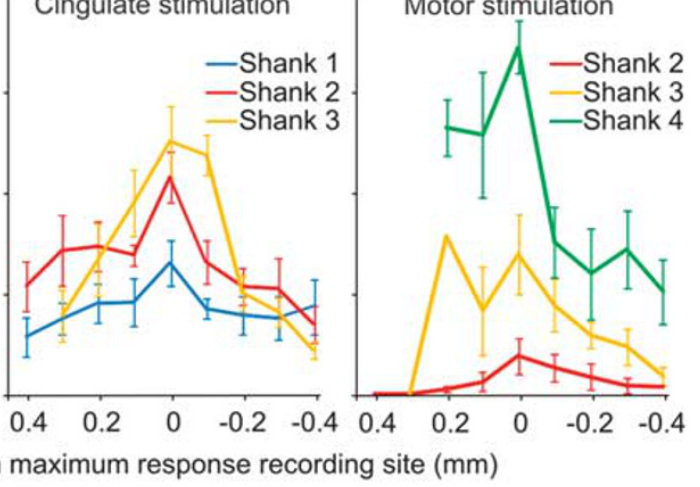

c

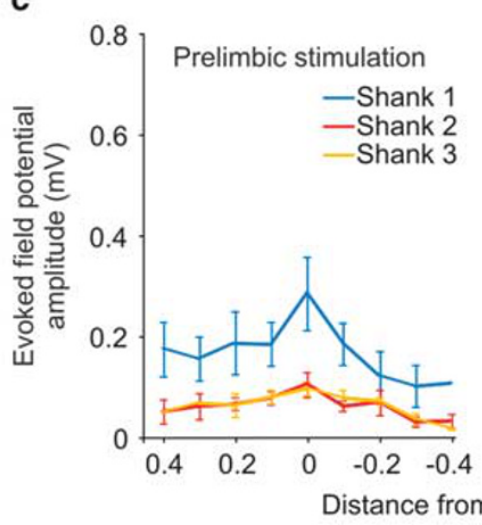

DAN lesion (5)

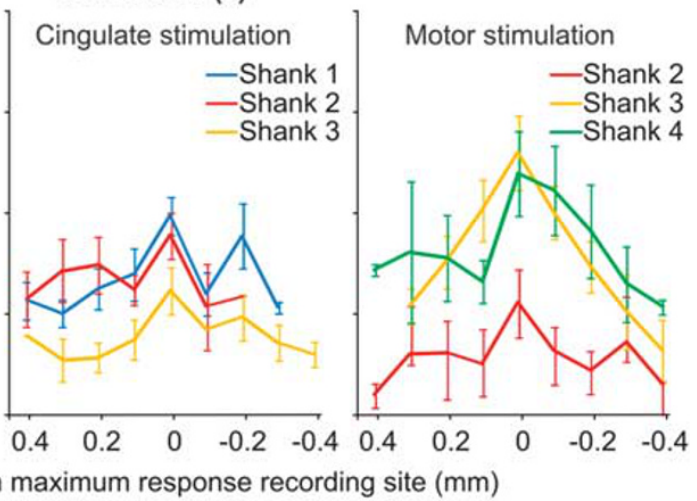

d

Maximum response recording site

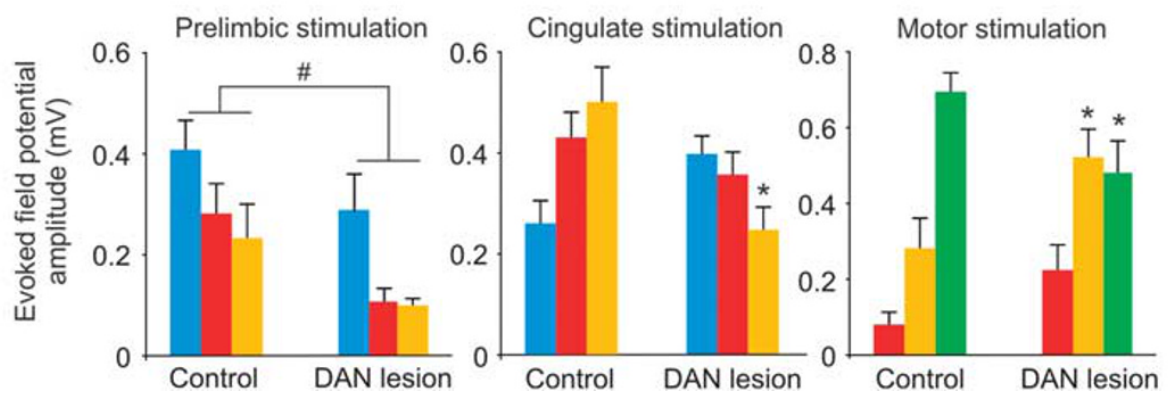

Figure 5 Disorganized response pattern to cortical inputs in dopamine neuron depleted mice. (a) Schematic diagram of the positioning of cortical stimulation electrodes and the striatal recording electrode (four shanks, eight channels per shank, channel separation: $100 \mu \mathrm{m}$ ). (b, c) Amplitude of the field potential responses evoked from prelimbic, cingulate, and motor cortex stimulation (700 $\mu \mathrm{A})$ in different striatal regions (shanks) in control (b) and DANlesioned mice (c). Responses of different animals were aligned at the site of maximal response within each shank. Positive distances are dorsal to the maximum. (d) Maximal amplitude of the response evoked at each shank by stimulation $(700 \mu \mathrm{A})$ of each cortical area in control and DAN-lesioned mice. In DAN-lesioned mice, the responses to prelimbic stimulation were reduced at all shanks and the pattern of responses to cingulate and motor stimulation was degraded (two-way ANOVAs, ${ }^{\#} P<0.05$ treatment main effect; $* P<0.05$ control vs DAN-lesioned, Tukey's post hoc test after significant treatment per shank interaction). Data are mean \pm SEM; animal numbers are indicated in parentheses.

completely impaired in DAN-lesioned mice. Although DANlesioned animals are usually outperformed by control animals, in some tasks they show no impairment (Levine et al, 1980) or perform better than controls by using novel strategies (Pearson et al, 1984). Moreover, they exhibit normal motivation (Moy, 1995; Pearson et al, 1984) and normal reward effectiveness in self-stimulation tasks (Takeichi et al, 1986; Stellar et al, 1988). Thus, no clear description of the core cognitive alteration underlying the learning deficits emerged from these studies (Moy, 1995), nor a hypothesis linking learning deficits to hyperactivity (Heffner and Seiden, 1983; Pearson et al, 1984). Deficient salience processing, resulting in reduced interest in exploiting and discovering, would very likely affect most kinds of learning irrespective of the neural substrates required by each specific task.

The hyperactivity of DAN-lesioned mice takes the form of different local exploratory behaviors adapted to each setting (ie, vertical exploration, swaying head movements, head dippings). However, in complex environments like the $\mathrm{Y}$ 
a

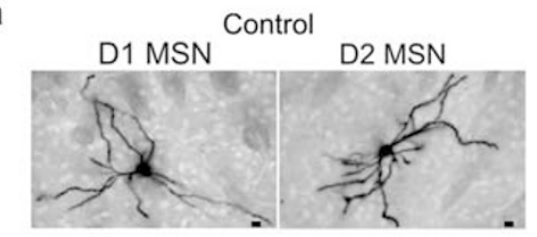

Control

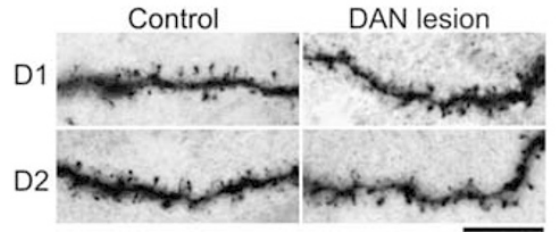

b

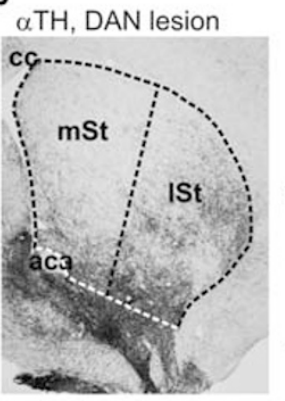

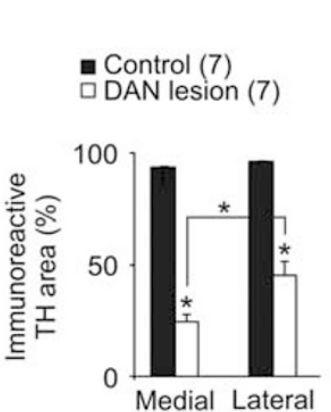

Medial Lateral

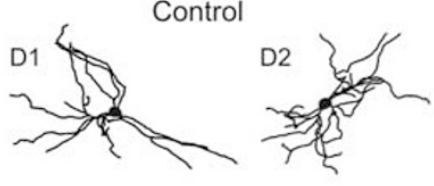

DAN lesion

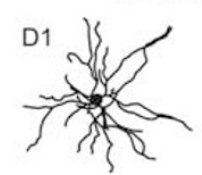

D2

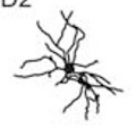

c

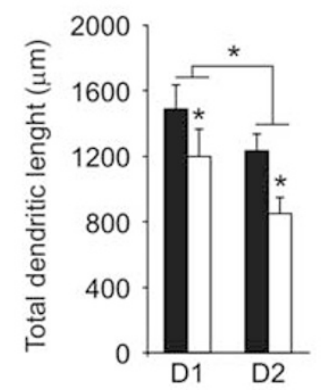

d
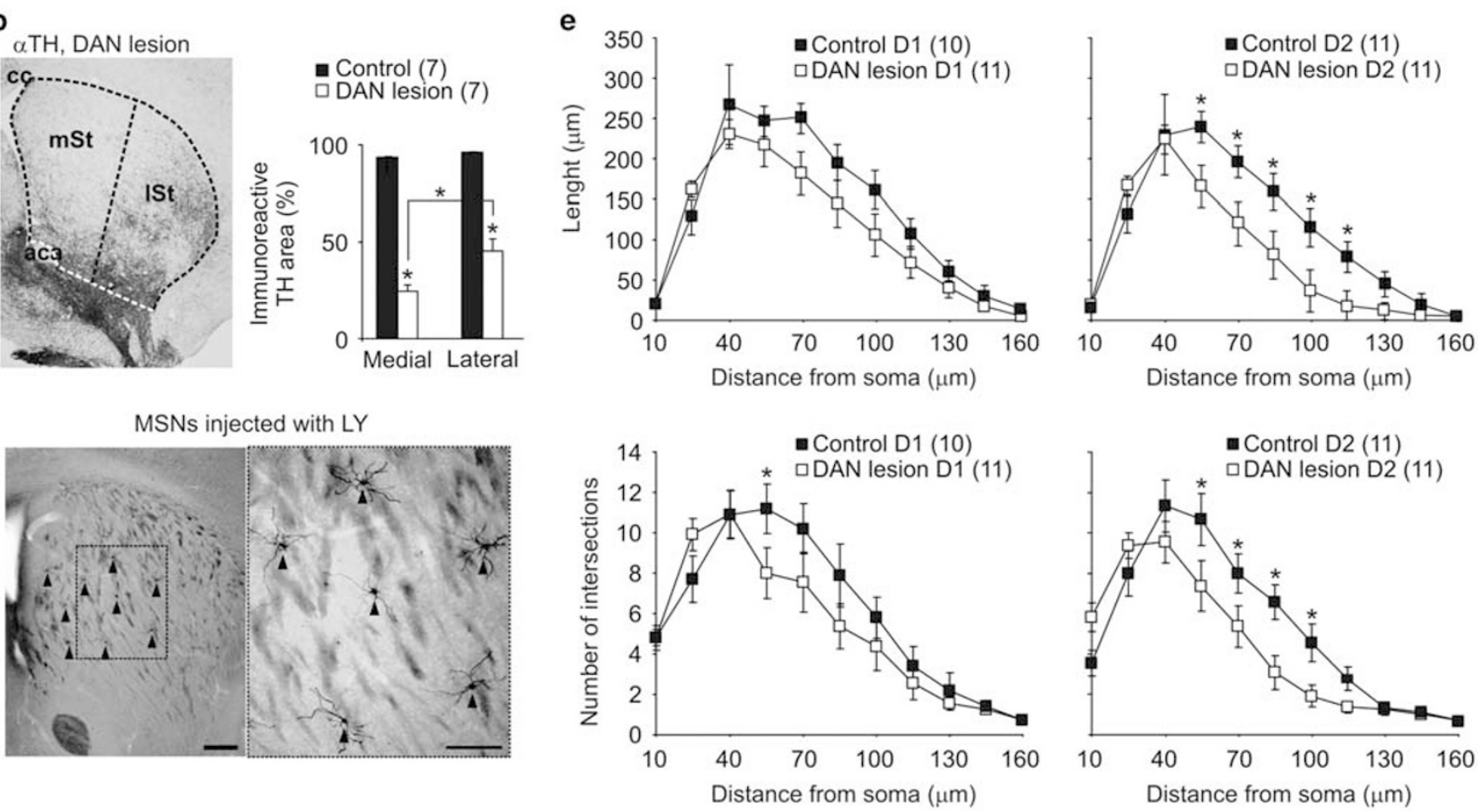

Figure 6 Contraction of medium spiny neuron dendritic fields in DAN-lesioned animals. (a) Photomicrograph of representative control direct and indirect pathway MSNs, high-magnification images of dendrites, and examples of reconstructions of the dendritic tree. (b) Striatal section immunostained for TH in representative DAN-depleted mice (aca, anterior commissure; mSt, medial striatum; ISt, lateral striatum; cc, corpus callosum). In DAN-lesioned mice, the percentage of immunoreactive TH area was lower in the mSt compared with the ISt (RM ANOVA, interaction: $F_{1,27}=17.1, P=0.00$ I; * $P<0.05$ Tukey's comparisons). Grid of MSNs injected with Lucifer yellow (LY) and immunostained with antibodies against the dye (right). (c, d) DAN-depleted mice showed a reduction in total dendritic length in both types of MSN (c: two-way ANOVA, treatment effect: $F_{1,42}=7.4, * P=0.009 ; M S N$ type effect: $F_{1,42}=6.0$, * $P=0.02$ ) without changes in spine density (d: two-way ANOVA). (e) Sholl analysis: dendritic length at different distances from the soma (upper panel) and number of dendritic branches intersecting circular rings drawn around the soma (lower panel). Distal dendritic length was reduced in iMSNs (RM ANOVA, interaction $\left.F_{10,230}=6.1, P<0.001\right)$ but not dMSNs $\left(F_{10,230}=1.5\right.$, NS), whereas intersections were reduced in both $\left(R M A N O V A\right.$ interactions, iMSNs: $F_{10,230}=3.9$, $P<0.001$ dMSNs: $\left.F_{10,230}=2.1, P=0.027\right)$. $* P<0.05$ Tukey's post hoc test after significant interaction). Data are mean \pm SEM; animal numbers are indicated in parentheses; scale bar: $10 \mu \mathrm{m}$ (a) and $100 \mu \mathrm{m}$ (b).

maze, where rodents display very specialized search routines, the total traveled distance is shorter in DAN-lesioned mice, ruling out a general form of hyperactivity in this model. We propose that DAN-lesioned mice are impaired at making transitions from local exploration to global search. The decision to engage in a costly search is driven by the possibility that richer resources will be found in other parts of the environment and is thought to depend on incentive signals carried by DA and other neuromodulators (Berridge and Robinson, 1998; Cohen et al, 2007; Adams et al, 2012; Dolan and Dayan, 2013). Likely, a deficiency in attributing salience to environments carrying high uncertainty would produce symptoms only in those environments, where less efficient exploratory behaviors would substitute for the required specialized search strategies.

The behavioral syndrome induced by neonatal DAN lesion is unlikely to be related to DA deficiency during behavioral testing. Acute DAR blockade in adults induces akinesia and rigidity instead of an exploration/exploitation imbalance. Moreover, neonatally DAN-lesioned mice are relatively insensitive to DAR antagonists (Duncan et al, 1987). Finally, preliminary data suggest that psychostimulants do not reverse the symptoms in adult DAN-lesioned mice (Supplementary Figure S4). On the other hand, DA has effects on 
corticostriatal plasticity that could shape corticostriatal connectivity (Kreitzer and Malenka, 2008; Surmeier et al, 2009) and could have long-lasting effects on behavior (Durston et al, 2005; Cohen et al, 2009). Previously, we found a delayed maturation of corticostriatal physiology in this animal model, but the activity of individual MSNs was almost normal in the adult (Galiñanes et al, 2009), contrasting with the marked behavioral alterations reported here. Indeed, we find that some behavioral alterations emerge or are exacerbated in adulthood. Adults use suboptimal search strategies in simple environments and show deficient alternation in the $\mathrm{Y}$ maze indicative of a working memory impairment, and these deficits are not present in juvenile mice. Here, by using local field potential recordings to more globally assess corticostriatal connectivity, we reveal a marked reduction of prefrontostriatal connectivity and a topographical disorganization of cingulostriatal projections in the adult. In addition, we found a putative morphological correlate of these alterations in the contraction of the dendritic arbor of iMSNs and, to a lesser degree, dMSNs. In contrast, we find more subtle alterations of prefrontostriatal connectivity in juvenile mice, in line with our previous study revealing a delayed maturation of corticostriatal synchronization in this model (Galiñanes et al, 2009). Importantly, prefrontostriatal connectivity may be crucial for working memory and decision making. Although prefrontal cortex contains the mechanisms maintaining working memory contents, basal ganglia circuits may be responsible for updating those contents (Chatham et al, 2014). Moreover, binary decisions like those involved in foraging behaviors are thought to be implemented by a striatal thresholding mechanism (Hayden et al, 2011; Ding and Gold, 2013). Thus, the persistence and aggravation of some symptoms in DAN-lesioned mice may partly result from progressive alterations in the connections linking associative frontal areas with the dorsomedial striatum.

At difference with nigrostriatal lesion induced in the adult, which reportedly produces spine depletion accompanied or not by dendritic shrinkage (Day et al, 2006; Villalba et al, 2009; Zhang et al, 2013; Suárez et al, 2014), MSNs do not show significant spine pruning in our neonatal lesion model. Although several factors can account for the observed differences (eg, lesion extent), early lesions could trigger compensations that are available only at certain developmental stages but not after the circuit has completed its maturation. Of note, in addition to direct effects of dopamine on spine formation (Fasano et al, 2013), complex network effects (Kozorovitskiy et al, 2012) and other factors expressed by DANs, like brain-derived neurotrophic factor, may differentially modulate corticostriatal connectivity along postnatal development. Thus, multiple mechanisms may contribute to the alterations of corticostriatal connectivity after early dopaminergic lesions.

There are several parallels between the behavioral alterations observed in DAN-lesioned mice and those of patients with ADHD, including setting-dependent hyperactivity and impaired working memory, social behavior, and learning. Moreover, patients with ADHD show altered functional connectivity between frontal association cortex and caudate nucleus and reduced caudate volume (Konrad and Eickhoff, 2010; Liston et al, 2011). However, it is not clear if an early hypodopaminergic state can account for the attention deficit and impulsivity that are crucial for ADHD diagnosis. Attention is regarded as a mechanism that optimizes information seeking (Gottlieb, 2012) and as a form of foraging (Manohar and Husain, 2013). Accordingly, 'exploratory attention' is guided by uncertainty, novelty, and surprise, whereas 'targeted attention' is guided by rewards. Thus, the salience and reward signals conveyed by DANs may have a role in attention (Redgrave and Gurney, 2006; Gottlieb, 2012). Some forms of impulsivity have also been related to delay aversion and DA modulation (Sagvolden et al, 2005; Sonuga-Barke, 2005). However, in ADHD the attention deficit and the impulsivity may relate to impaired behavioral inhibition and altered noradrenergic modulation of the prefrontal cortex (Castellanos and Tannock, 2002; Arnsten, 2011). Our findings suggest that ADHD and other neuropsychiatric conditions presumably linked to developmental alterations of the dopaminergic system should be examined for alterations in foraging decision making.

In summary, deficits in foraging decision making and alterations of frontostriatal connectivity emerge as candidate intermediate phenotypes for deficient DAN function beginning in early life.

\section{FUNDING AND DISCLOSURE}

This study was supported by grants from FONCYT (PICT2008-2205, PICT2011-1679, and PICT2011-521), University of Buenos Aires (UBACYT-2011-562 and 2014249), and CONICET (PIP077). The authors declare no conflict of interest.

\section{ACKNOWLEDGMENTS}

The authors thank Graciela Ortega for excellent technical assistance.

\section{REFERENCES}

Adams GK, Watson KK, Pearson J, Platt ML (2012). Neuroethology of decision-making. Curr Opin Neurobiol 22: 982-989.

Archer T, Danysz W, Fredriksson A, Jonsson G, Luthman J, Sundström E et al (1988). Neonatal 6-hydroxydopamine-induced dopamine depletions: motor activity and performance in maze learning. Pharmacol Biochem Behav 31: 357-364.

Arnsten AFT (2011). Catecholamine influences on dorsolateral prefrontal cortical networks. Biol Psychiatry 69: e89-e99.

Avale ME, Falzone TL, Gelman DM, Low MJ, Grandy DK, Rubinstein M (2004). The dopamine D4 receptor is essential for hyperactivity and impaired behavioral inhibition in a mouse model of attention deficit/hyperactivity disorder. Mol Psychiatry 9: 718-726.

Belforte JE, Zsiros V, Sklar ER, Jiang Z, Yu G, Li Y et al (2010). Postnatal NMDA receptor ablation in corticolimbic interneurons confers schizophrenia-like phenotypes. Nat Neurosci 13: 76-83.

Berridge KC, Robinson TE (1998). What is the role of dopamine in reward: hedonic impact, reward learning, or incentive salience? Brain Res Brain Res Rev 28: 309-369.

Biederman J, Faraone S V (2005). Attention-deficit hyperactivity disorder. Lancet 366: 237-248.

Bromberg-Martin ES, Hikosaka O (2009). Midbrain dopamine neurons signal preference for advance information about upcoming rewards. Neuron 63: 119-126. 
Castellanos FX, Tannock R (2002). Neuroscience of attentiondeficit/hyperactivity disorder: the search for endophenotypes. Nat Rev Neurosci 3: 617-628.

Chatham CH, Frank MJ, Badre D (2014). Corticostriatal output gating during selection from working memory. Neuron 81: 930-942.

Cohen JD, McClure SM, Yu AJ (2007). Should I stay or should I go? How the human brain manages the trade-off between exploitation and exploration. Philos Trans R Soc Lond B Biol Sci 362: 933-942.

Cohen MX, Schoene-Bake J-C, Elger CE, Weber B (2009). Connectivity-based segregation of the human striatum predicts personality characteristics. Nat Neurosci 12: 32-34.

Daw ND, O'Doherty JP, Dayan P, Seymour B, Dolan RJ (2006). Cortical substrates for exploratory decisions in humans. Nature 441: 876-879.

Day $M$, Wang Z, Ding J, An X, Ingham C A, Shering AF et al (2006). Selective elimination of glutamatergic synapses on striatopallidal neurons in Parkinson disease models. Nat Neurosci 9: 251-259.

Deacon R (2012). Assessing burrowing, nest construction, and hoarding in mice. $J$ Vis Exp 59: e2607.

Ding L, Gold JI (2013). The basal ganglia's contributions to perceptual decision making. Neuron 79: 640-649.

Dolan RJ, Dayan P (2013). Goals and habits in the brain. Neuron 80: 312-325.

Duncan GE, Criswell HE, McCown TJ, Paul IA, Mueller RA, Breese GR (1987). Behavioral and neurochemical responses to haloperidol and SCH-23390 in rats treated neonatally or as adults with 6-hydroxydopamine. J Pharmacol Exp Ther 243: 1027-1034.

Durston S, Fossella JA, Casey BJ, Hulshoff Pol HE, Galvan A, Schnack HG et al (2005). Differential effects of DRD4 and DAT1 genotype on fronto-striatal gray matter volumes in a sample of subjects with attention deficit hyperactivity disorder, their unaffected siblings, and controls. Mol Psychiatry 10: 678-685.

Fasano C, Bourque M-J, Lapointe G, Leo D, Thibault D, Haber M et al (2013). Dopamine facilitates dendritic spine formation by cultured striatal medium spiny neurons through both D1 and D2 dopamine receptors. Neuropharmacology 67: 432-443.

Galiñanes GL, Braz BY, Murer MG (2011). Origin and properties of striatal local field potential responses to cortical stimulation: temporal regulation by fast inhibitory connections. PLoS One 6: e28473.

Galiñanes GL, Taravini IRE, Murer MG (2009). Dopaminedependent periadolescent maturation of corticostriatal functional connectivity in mouse. J Neurosci 29: 2496-2509.

Gottlieb J (2012). Attention, learning, and the value of information. Neuron 76: 281-295.

Haber SN, Knutson B (2010). The reward circuit: linking primate anatomy and human imaging. Neuropsychopharmacology 35: $4-26$.

Hayden BY, Pearson JM, Platt ML (2011). Neuronal basis of sequential foraging decisions in a patchy environment. Nat Neurosci 14: 933-939.

Heffner TG, Seiden LS (1983). Impaired acquisition of an operant response in young rats depleted of brain dopamine in neonatal life. Psychopharmacology (Berl) 79: 115-119.

Kasanetz F, Riquelme LA, Della-Maggiore V, O’Donnell P, Murer MG (2008). Functional integration across a gradient of corticostriatal channels controls UP state transitions in the dorsal striatum. Proc Natl Acad Sci U S A 105: 8124-8129.

Klein RG, Mannuzza S, Olazagasti MAR, Roizen E, Hutchison JA, Lashua EC et al (2012). Clinical and functional outcome of childhood attention-deficit/hyperactivity disorder 33 years later. Arch Gen Psychiatry 69: 1295-1303.

Konrad K, Eickhoff SB (2010). Is the ADHD brain wired differently? A review on structural and functional connectivity in attention deficit hyperactivity disorder. Hum Brain Mapp 31: 904-916.
Kozorovitskiy Y, Saunders A, Johnson CA, Lowell BB, Sabatini BL (2012). Recurrent network activity drives striatal synaptogenesis. Nature 485: 646-650.

Kreitzer AC, Malenka RC (2008). Striatal plasticity and basal ganglia circuit function. Neuron 60: 543-554.

Levine TE, Erinoff L, Dregits DP, Seiden LS (1980). Effects of neonatal and adult 6-hydroxydopamine treatment on randominterval behavior. Pharmacol Biochem Behav 12: 281-285.

Liston C, Malter Cohen M, Teslovich T, Levenson D, Casey BJ (2011). Atypical prefrontal connectivity in attention-deficit/ hyperactivity disorder: pathway to disease or pathological end point? Biol Psychiatry 69: 1168-1177.

Luthman J, Bassen M, Fredriksson A, Archer T (1997). Functional changes induced by neonatal cerebral 6-hydroxydopamine treatment: effects of dose levels on behavioral parameters. Behav Brain Res 82: 213-221.

Mailly P, Aliane V, Groenewegen HJ, Haber SN, Deniau J-M (2013). The rat prefrontostriatal system analyzed in 3D: evidence for multiple interacting functional units. J Neurosci 33: 5718-5727.

Manohar SG, Husain M (2013). Attention as foraging for information and value. Front Hum Neurosci 7: 711.

Miller FE, Heffner TG, Kotake C, Seiden LS (1981). Magnitude and duration of hyperactivity following neonatal 6-hydroxydopamine is related to the extent of brain dopamine depletion. Brain Res 229: 123-132.

Moy SS (1995). Impaired acquisition and operant responding after neonatal dopamine depletion in rats. Pharmacol Biochem Behav 52: 433-441.

Pearson DE, Raskin LA, Shaywitz BA, Anderson GM, Cohen DJ (1984). Radial arm maze performance in rats following neonatal dopamine depletion. Dev Psychobiol 17: 505-517.

Pearson JM, Platt ML (2013). Dopamine: burning the candle at both ends. Neuron 79: 831-833.

Redgrave P, Gurney K (2006). The short-latency dopamine signal: a role in discovering novel actions? Nat Rev Neurosci 7: 967-975.

Sagvolden T, Johansen EB, Aase H, Russell VA (2005). A dynamic developmental theory of attention-deficit/hyperactivity disorder (ADHD) predominantly hyperactive/impulsive and combined subtypes. Behav Brain Sci 28: 397-419 discussion 419-468.

Shaywitz BA, Yager RD, Klopper JH (1976). Selective brain dopamine depletion in developing rats: an experimental model of minimal brain dysfunction. Science 191: 305-308.

Shuen JA, Chen M, Gloss B, Calakos N (2008). Drd1a-tdTomato BAC transgenic mice for simultaneous visualization of medium spiny neurons in the direct and indirect pathways of the basal ganglia. J Neurosci 28: 2681-2685.

Smith RD, Cooper BR, Breese GR (1973). Growth and behavioral changes in developing rats treated intracisternally with 6hydroxydopamine: evidence for involvement of brain dopamine. J Pharmacol Exp Ther 185: 609-619.

Sonuga-Barke EJS (2005). Causal models of attention-deficit/ hyperactivity disorder: from common simple deficits to multiple developmental pathways. Biol Psychiatry 57: 1231-1238.

Stellar JR, Waraczynski M, Bruno JP (1988). Neonatal dopamine depletions spare lateral hypothalamic stimulation reward in adult rats. Pharmacol Biochem Behav 30: 365-370.

Surmeier DJ, Plotkin J, Shen W (2009). Dopamine and synaptic plasticity in dorsal striatal circuits controlling action selection. Curr Opin Neurobiol 19: 621-628.

Suárez LM, Solís O, Caramés JM, Taravini IR, Solís JM, Murer MG et al (2014). L-DOPA treatment selectively restores spine density in dopamine receptor D2-expressing projection neurons in dyskinetic mice. Biol Psychiatry 75: 711-722.

Swanson JM, Kinsbourne M, Nigg J, Lanphear B, Stefanatos GA, Volkow N et al (2007). Etiologic subtypes of attention-deficit/ hyperactivity disorder: brain imaging, molecular genetic and environmental factors and the dopamine hypothesis. Neuropsychol Rev 17: 39-59. 
Takeichi T, Kurumiya S, Umemoto M, Olds ME (1986). Roles of catecholamine terminals and intrinsic neurons of the ventral tegmentum in self-stimulation investigated in neonatally dopamine-depleted rats. Pharmacol Biochem Behav 24: 1101-1109.

Toga AW, Thompson PM, Sowell ER (2006). Mapping brain maturation. Trends Neurosci 29: 148-159.

Villalba RM, Lee H, Smith Y (2009). Dopaminergic denervation and spine loss in the striatum of MPTP-treated monkeys. Exp Neurol 215: 220-227.

Volkow ND, Wang G-J, Newcorn J, Telang F, Solanto MV, Fowler JS et al (2007). Depressed dopamine activity in caudate and preliminary evidence of limbic involvement in adults with attention-deficit/hyperactivity disorder. Arch Gen Psychiatry 64: 932-940.

Wilens TE, Biederman J, Faraone SV, Martelon M, Westerberg D, Spencer TJ (2009). Presenting ADHD symptoms, subtypes, and comorbid disorders in clinically referred adults with ADHD. J Clin Psychiatry 70: 1557-1562.

Zhang Y, Meredith GE, Mendoza-Elias N, Rademacher DJ, Tseng KY, Steece-Collier K (2013). Aberrant restoration of spines and their synapses in L-DOPA-induced dyskinesia: involvement of corticostriatal but not thalamostriatal synapses. J Neurosci 33: 11655-11667.

Supplementary Information accompanies the paper on the Neuropsychopharmacology website (http://www.nature.com/npp) 